\title{
Humidity, Pressure, and Temperature Measurements in an Interdigitated-Flow PEM Hydrogen Fuel Cell
}

\author{
S. Bell · G. Hinds - M. de Podesta • \\ M. Stevens • J. Wilkinson
}

Received: 26 August 2010 / Accepted: 8 September 2011 / Published online: 24 September 2011

(C) Crown copyright 2011

\begin{abstract}
In situ measurements of humidity, temperature, and pressure are demonstrated for a polymer electrolyte membrane (PEM) fuel cell of interdigitated gas flow channel layout. Sensors are embedded at the flowfield edges, sampling air, and hydrogen at several positions along the flow channels. The measurements provide, for the first time, real-time localized data for this configuration of fuel cell. The results show that, for the interdigitated flowfield, moisture freely permeates through the membrane electrode assembly (MEA). This is in contrast to lower net moisture transmission through the same MEA used in a serpentine flowfield.
\end{abstract}

Keywords Humidity · In situ measurement - PEM fuel cell · Pressure - Temperature

\section{Introduction}

Hydrogen fuel cells have great potential as portable sources of power that are pollution-free at the point of use, and can be low-carbon if the fuel (hydrogen) is generated by renewable methods. However, the reliability and durability of fuel cells need to be improved significantly if this technology is to be widely adopted. Hence, there is considerable interest in research that can improve the performance and lifespan of the critical component - the membrane electrode assembly (MEA). Testing under controlled conditions is a necessary step towards viability and uptake of fuel cells. Detailed measurement data are also needed as inputs to models of fuel cell operation.

C Crown copyright 2010. Reproduced by permission of the Controller of HMSO and the Queen's printer for Scotland.

S. Bell $(\varangle) \cdot$ G. Hinds $\cdot$ M. de Podesta $\cdot$ M. Stevens $\cdot$ J. Wilkinson National Physical Laboratory, Teddington, Middlesex TW11 0LW, UK e-mail: stephanie.bell@npl.co.uk 
Yet, in spite of widespread interest, suitable measurement techniques are still only in the process of being developed.

There is a strong research focus on low-temperature polymer electrolyte membrane (PEM) fuel cells. Measurements of the reactant gas humidity, temperature, flow, pressure, and composition are central to the study of PEM cells, along with electrical measurements. Water is a product of the fuel cell reaction. The presence of water within the fuel cell membrane usefully increases its proton conductivity. However, if conditions are too wet, with liquid water blocking microscopic pores in the electrodes, then transport of the reactant gases to the catalyst surface is impeded. If too dry, membrane conductivity worsens. For these reasons, it is highly important to measure and control humidity in PEM fuel cell gases. Temperature measurements are also essential: not only does temperature influence cell operation, but localized overheating is an indicator of uneven current generation and a potential cause of cell degradation. All these effects can be studied if localized values of temperature and humidity can be correlated with localized current density within the cell. In support of this, localized pressure and flow measurements are also valuable.

In situ humidity measurements in this application are difficult because of the inaccessibility and small size of the flow channels. The conditions of temperature up to $90{ }^{\circ} \mathrm{C}$ and humidity up to saturation can be challenging for some measurement methods. In addition, sensors can be at risk of drift due to chemical interferences or permanent contamination. In recent years the distribution of liquid water in PEM fuel cells has been studied using neutron radiography, as reviewed recently [1]. For drier operating conditions such as those of relevance to automotive applications, measurement of relative humidity is more applicable. A number of different techniques have been investigated including gas chromatography [2], mass spectrometry through fine glass capillaries [3], fiber optic coupled absorption spectroscopy [4], and ex situ humidity sensing [5]. While these approaches all yield useful information about the variation in water content in the gas channels, they do not achieve the real-time localized measurements that are needed for an effective study.

Recently, NPL reported real-time localized measurements inside a functioning serpentine-flow fuel cell, using miniature temperature and relative humidity sensors embedded in the flowplates [6]. In the present work, a similar study is made on an interdigitated-flow fuel cell. The work reported here builds on and extends previous work, by studying a different configuration of fuel cell flow, and by observing a difference in the MEA characteristic in the two cases. Trial in situ pressure measurement is also reported for the first time.

\section{Measurements}

\subsection{Experimental Setup}

\subsubsection{Fuel Cell}

A schematic diagram of a cross section of the fuel cell is shown in Fig. 1. The membrane is a proton-conducting and electronically insulating polymer known as Nafion, 


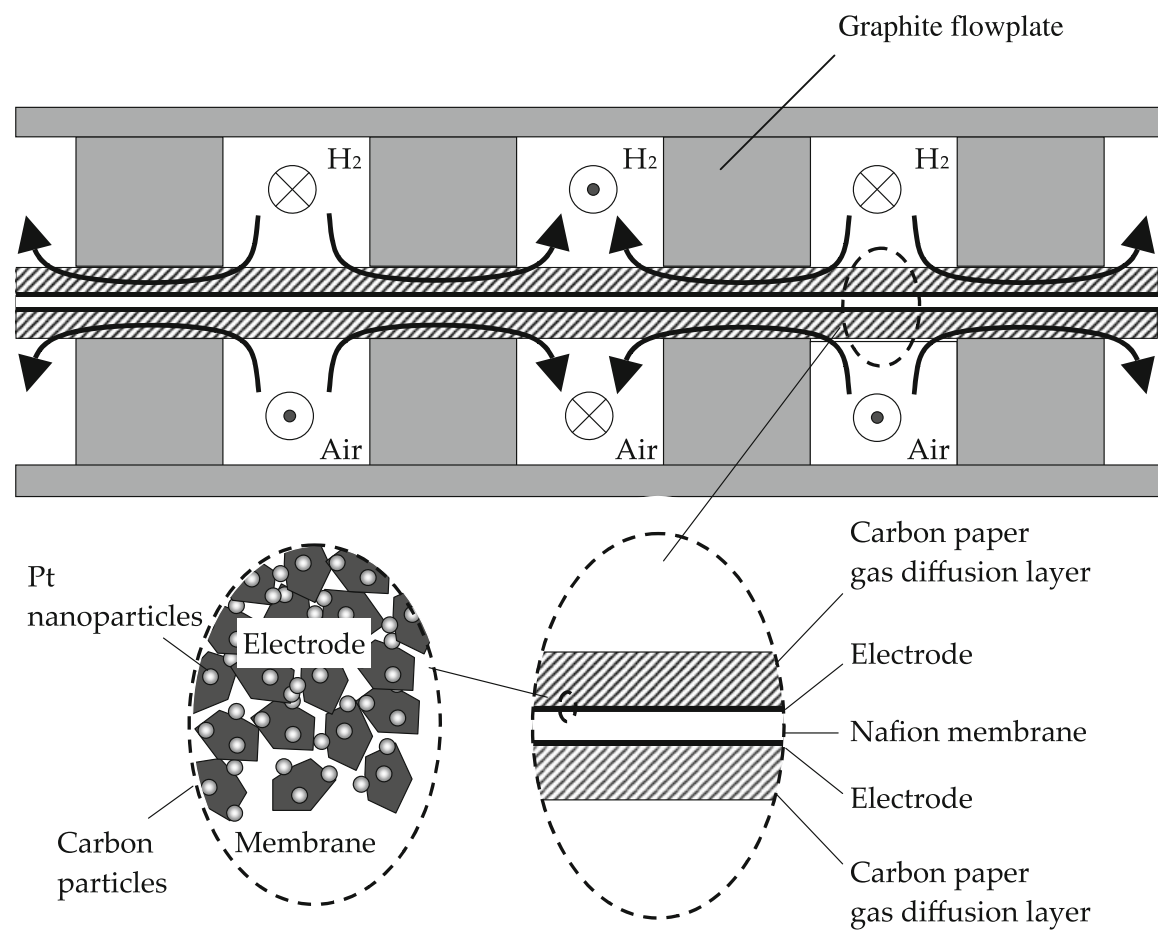

Fig. 1 Cross-sectional view of interdigitated-flow fuel cell with expanded views of MEA and electrode structures. Symbols $\odot$ and $\bigotimes$ represent gas flows, respectively, toward and away from the viewer. Curved arrows indicate gas flow via the gas diffusion layer structure (not shown to scale)

which consists of a hydrophobic PTFE backbone and hydrophilic sulphonic acid side chains. The proton conductivity of the hydrophilic side chains increases with water content in the membrane. On each side of the membrane are two thin porous electrodes (typically $10 \mu \mathrm{m}$ thick), in which the electrochemical reactions take place. At the anode, hydrogen gas is reduced to protons and electrons. The protons migrate through the membrane to the cathode under the influence of the electric field, while the electrons are forced to flow around an external circuit, generating useful power. At the cathode, the protons and electrons combine with oxygen atoms to form water and heat. The electrodes consist of Pt nanoparticles (diameter of $3 \mathrm{~nm}$ to $5 \mathrm{~nm}$ ) supported on high surface area carbon particles (diameter $\sim 30 \mathrm{~nm}$ ) and mixed with Nafion to form a "three phase" structure. The power density of the electrode depends on the number of electrochemically active sites (where the boundaries of the three phases coincide) and their connectivity to the respective bulk phases.

Gas is supplied to each electrode through a graphite flow plate and a sheet of carbon paper known as a gas diffusion layer (GDL). The purpose of these components is to distribute the gas evenly over the active area of the fuel cell, while simultaneously collecting or distributing current to the electrodes. Another important function of the GDL is to facilitate removal of the water formed at the cathode in the electrochemical 


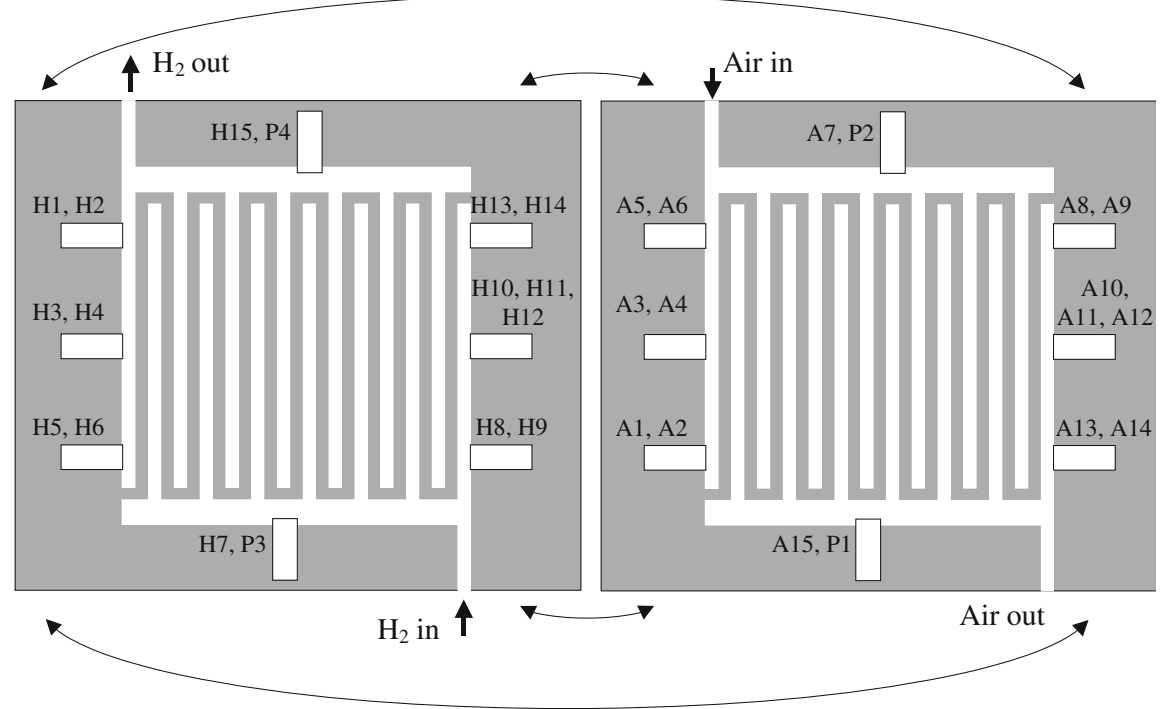

Fig. 2 Schematic diagram showing interdigitated flowfield layout, which is symmetrical between the hydrogen and air sides of the fuel cell. For clarity, only seven inlet and seven outlet channels are illustrated, but in fact there were 17 inlet and 17 outlet channels. Locations of combined humidity and temperature sensors are shown (H1 to H15 and A1 to A15), as well as pressure sensors P1 to P4. The assembled plates face each other with sensors $\mathrm{H} 1$ and $\mathrm{H} 2$ opposite sensors A8 and A9 across the MEA. The inlet channels face each other in counter-flow, as do the outlet channels

reaction, so as to prevent blockage of the gas pores. For this reason the GDL is often treated with a hydrophobic agent such as PTFE.

The fuel cell used in this study comprised flowplates machined from impregnated graphite, and an MEA consisting of two identical Johnson Matthey Pt/C electrodes (Pt loading $0.4 \mathrm{mg} \cdot \mathrm{cm}^{-2}$ ) on a Nafion 115 membrane (thickness $127 \mu \mathrm{m}$ ). A Hydrogenics Test Systems FCATS-G50 test stand was used to control the fuel cell operation. The active area of the fuel cell was $7 \mathrm{~cm} \times 7 \mathrm{~cm}$ with individual channels of cross section approximately $1 \mathrm{~mm} \times 1 \mathrm{~mm}$. The flow field consisted of two interdigitated sets of flow channels, each traversing almost the full length of the active area. The inlet flow channels interleave with outlet channels but they are not directly connected. Gas crosses from inlet to outlet channels exclusively by forced flow through the GDL, bridging the barrier between channels along regions where the GDL is clamped by the plates as shown in Fig. 1. While the electrochemical reactions proceed at the electrodes, current is drawn via gold-plated stainless steel current collectors attached to the graphite plates. Figure 2 illustrates the interdigitated flowfield layout, which is symmetrical between the hydrogen and air sides of the assembly.

\subsubsection{Temperature, Pressure, and Humidity Sensors}

The temperature and humidity sensors used in the work were Sensirion SHT75 single chip combined sensors as used and characterized in previous work [6,7]. Each 
incorporates a bandgap temperature-sensing element and a capacitive polymer sensing element for relative humidity. Both sensors are coupled on-chip to a 14-bit analog-to-digital converter and a serial interface circuit, giving high signal quality, fast response time, and insensitivity to external disturbances. The miniature strain gauge pressure sensors were Precision Measurement Company Models 105S and 060S, with three-wire connections. These sensors were of two sizes: respectively nominally $3 \mathrm{~mm}$ in diameter and $1 \mathrm{~mm}$ thick; and nominally $1.5 \mathrm{~mm}$ in diameter and $0.5 \mathrm{~mm}$ thick.

The temperature and humidity sensors were calibrated in their embedded positions in the fuel cell plates in a temperature- and humidity-controlled chamber at approximately $80^{\circ} \mathrm{C}$. The calibration was performed in air at atmospheric pressure. The reference temperature was measured using calibrated platinum resistance thermometers placed close to the plates, and the reference humidity was measured by sampling gas from near the plates using a calibrated optical dew-point hygrometer. The embedded pressure sensors were calibrated against a calibrated pressure gauge in an assembled fuel cell at $300 \mathrm{kPa}$ (2 bar gauge) and $80^{\circ} \mathrm{C}$. Calibration corrections were evaluated for every humidity, temperature, and pressure sensor, and were subsequently applied to the sensor readings.

\subsubsection{Sensor Mounting}

As shown in Fig. 2, each flow plate was fitted with 15 combined humidity and temperature sensors located in pairs around the edge of the plate. Additionally, miniature pressure sensors were located in each plate, one upstream, one downstream (four in total).

The sensors were embedded in recesses along the edge of the channels as shown in the photograph of Fig. 3. In operation, a proportion of gas can bypass the channel, reaching the sensors. This allowed direct measurement of the temperature pressure and humidity of the gas in the narrow $1 \mathrm{~mm}^{2}$ channels, without significantly impeding the main flow. Previous work [6,7] with fuel cells of the same scale but different channel layout (serpentine flowfield) has demonstrated that the presence of the sensors and recesses does not significantly perturb the temperature and humidity conditions or the general operation of the fuel cell.

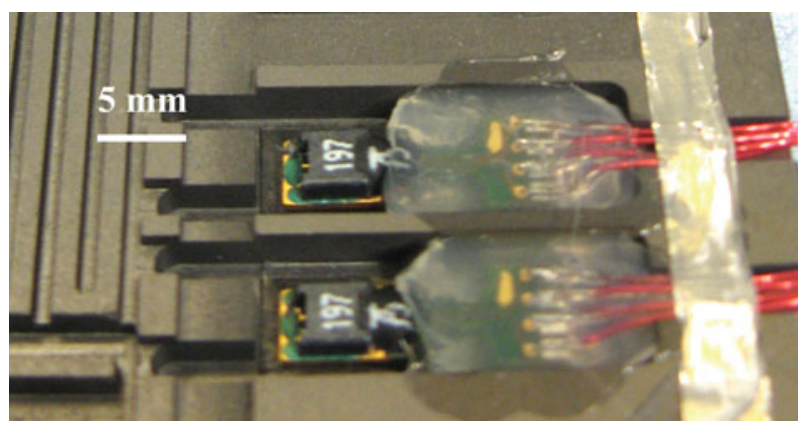

Fig. 3 Photograph of a pair of combined humidity and temperature sensors during installation in a recess alongside a fuel cell flow channel 


\subsection{Measurement Procedure}

The fuel cell assembly was mounted between stainless steel fixing plates, in which cartridge heaters were embedded to control the cell temperature. The gas path was helium leak-tested to ensure there were no leaks across or around the membrane. The cathode and anode were supplied with air and hydrogen humidified by bubbling though liquid water in saturators held at a controlled, adjustable temperature. The connecting pipework was heated to approximately $10^{\circ} \mathrm{C}$ above the cell temperature to prevent condensation. The fuel cell was oriented horizontally to avoid build-up of condensed water in the recesses containing the sensors. The MEA was positioned with the cathode facing downwards to facilitate draining of any liquid water generated in the fuel cell reaction. For a given inlet humidity, the current drawn from the cell was varied and the resulting humidities and temperatures along the flow channels were logged. The operating conditions that were maintained constant during the tests are listed in Table 1 . A relatively low inlet humidity was used (dew point of $60^{\circ} \mathrm{C}$ ) to highlight the observable changes in gas humidity on transit through the cell.

\section{Results}

Values of the dew-point temperature calculated from locally measured values of the relative humidity and temperature are shown in Fig. 4. Data from sensors on each flow plate are shown; those on cathode and anode inlet channels in Fig. 4a, and outlet channels in Fig. 4b. The graphs show $10 \mathrm{~h}$ of observations, with a current of $5 \mathrm{~A}$ drawn from $2.5 \mathrm{~h}$ onward. The numbers on the figures indicate the sensor number according to Fig. 2. The expanded uncertainty of dew-point values, based on relative humidity and temperature measurements including calibration uncertainty and typical stability of the sensors [7], was estimated to be $1.7^{\circ} \mathrm{C}$. Not every sensor is shown in Fig. 4: A8 and H6 appeared to fail, giving no results. Sensor A11 readings closely overlapped those of nearby A10 and A12, and are omitted for clarity. A7, A15, H7, and H15 gave results consistent with the others shown in Fig. 4, but over smaller ranges (partial

Table 1 Operating conditions maintained constant during the tests

\begin{tabular}{ll}
\hline Condition & Setting \\
\hline Cell temperature & $80^{\circ} \mathrm{C}$ \\
Cathode inlet gas temperature & $90^{\circ} \mathrm{C}$ \\
Anode inlet gas temperature & $90{ }^{\circ} \mathrm{C}$ \\
Air flow rate & $0.81 \mathrm{~L} \cdot \mathrm{min}^{-1}$ \\
$\mathrm{H}_{2}$ flow rate & $0.34 \mathrm{~L} \cdot \mathrm{min}^{-1}$ \\
Cathode inlet pressure & \\
Anode inlet pressure & All nominally $300 \mathrm{kPa}(2$ bar gauge $)$ \\
Cathode outlet pressure & \\
Anode outlet pressure & \\
\hline
\end{tabular}


(a)
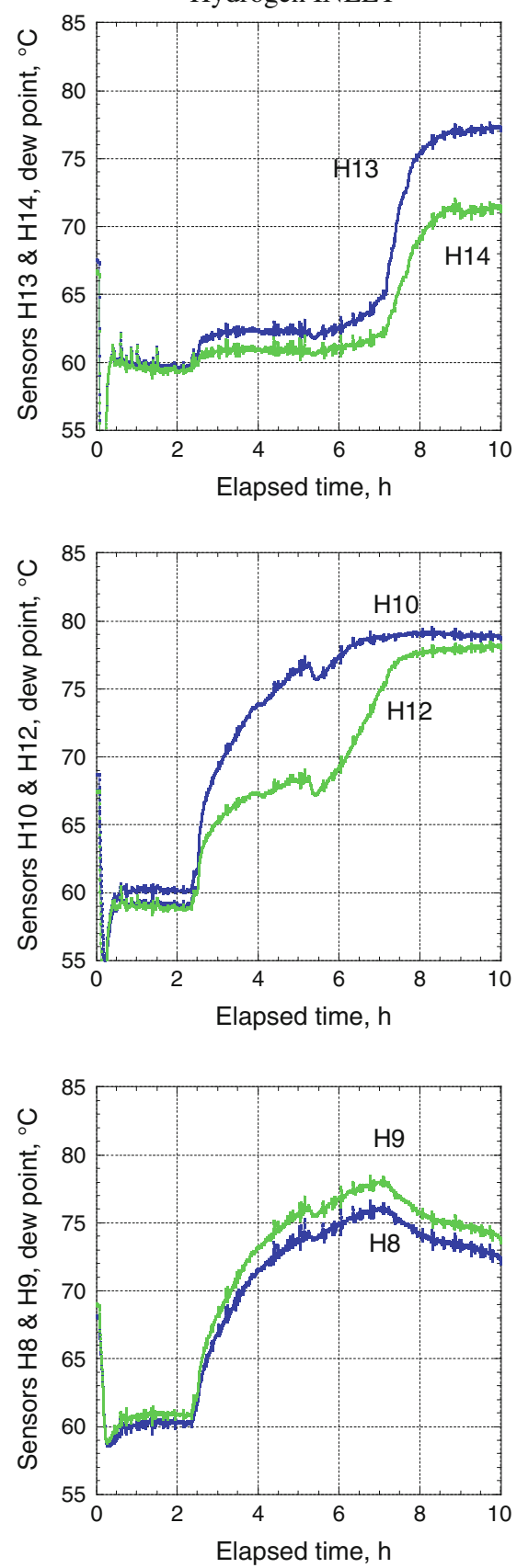
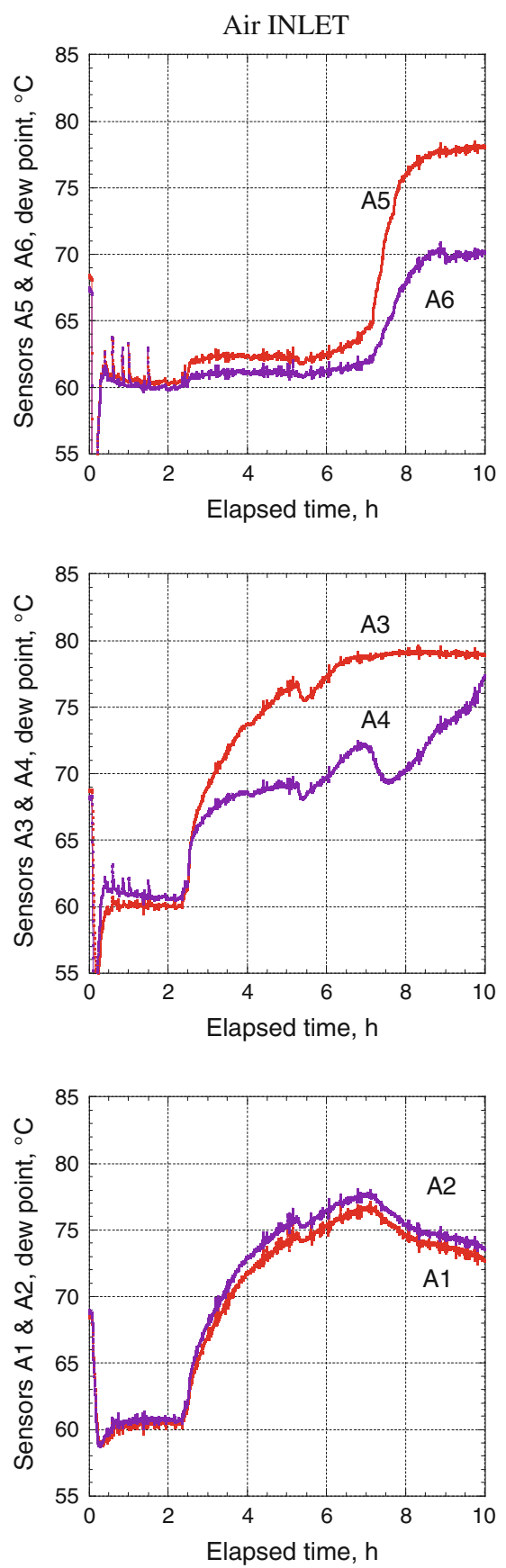

Fig. 4 Graphs showing localized dew point in fuel cell channels over a period of $10 \mathrm{~h}$, with current generation initiated at approximately $2.5 \mathrm{~h}$. Graphs are grouped by (a) hydrogen and air inlets and (b) hydrogen and air outlets. Graphs shown side by side correspond to sensors that face each other across the membrane (e.g., sensor H13 faces sensor A5) 
(b)
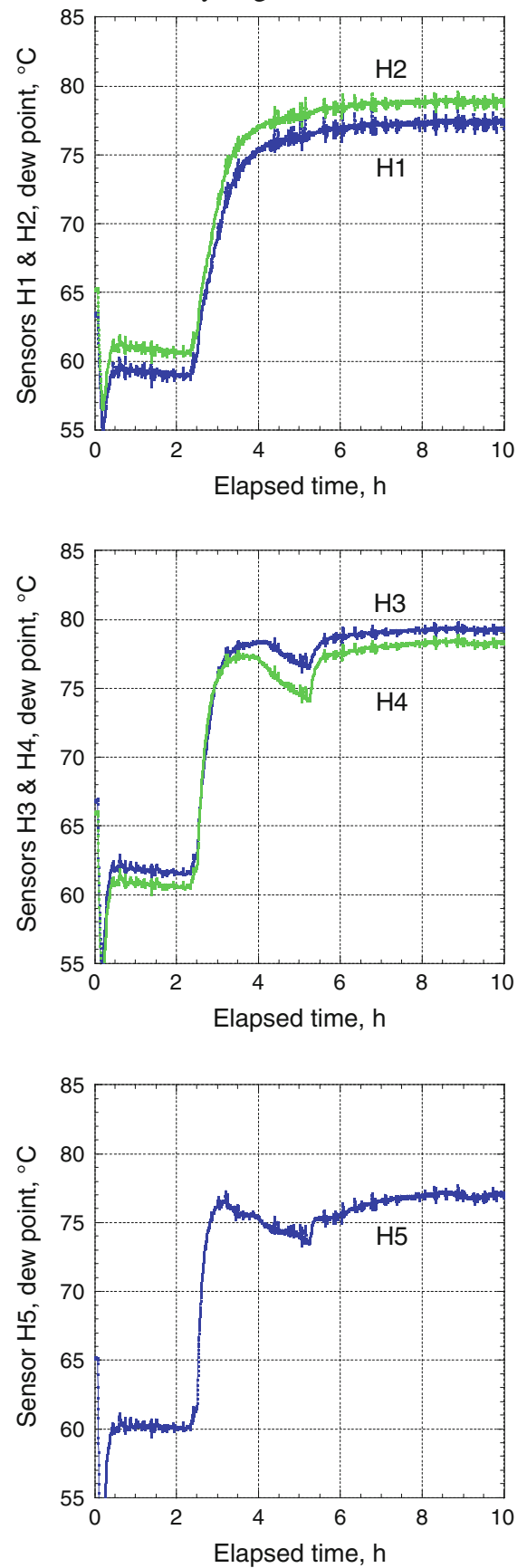

Fig. 4 continued
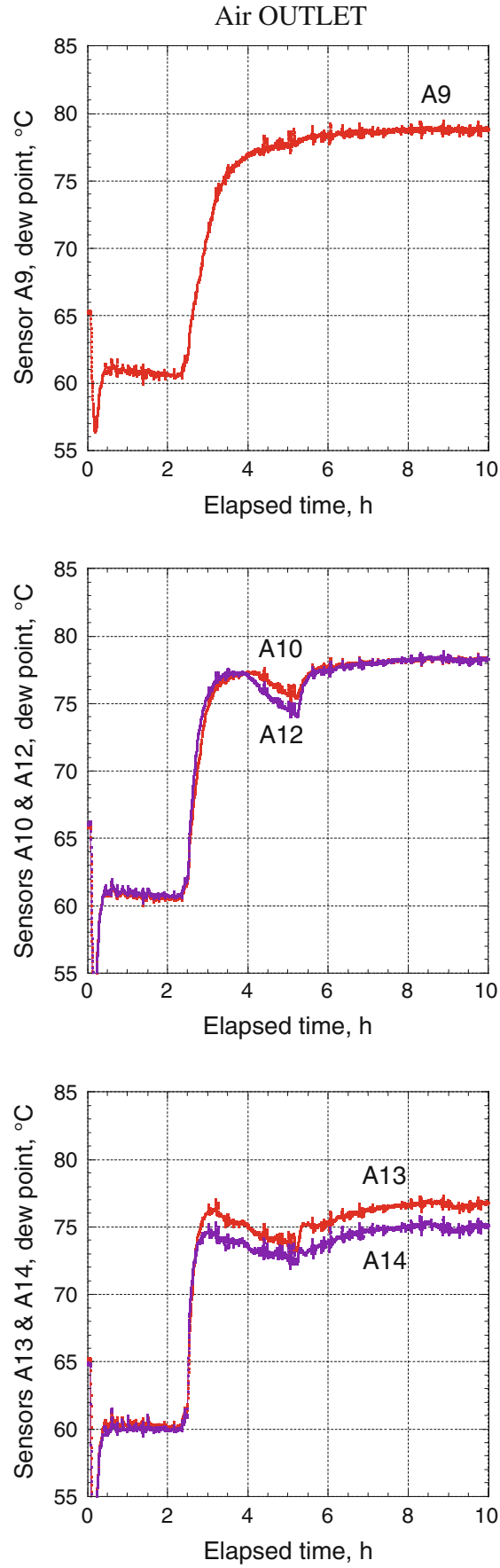
Fig. 5 Graph showing example of response time of facing sensors $\mathrm{H} 7$ and A15 to rising humidity at start of current generation. Data logging of sensors was synchronous to approximately $2 \mathrm{~s}$

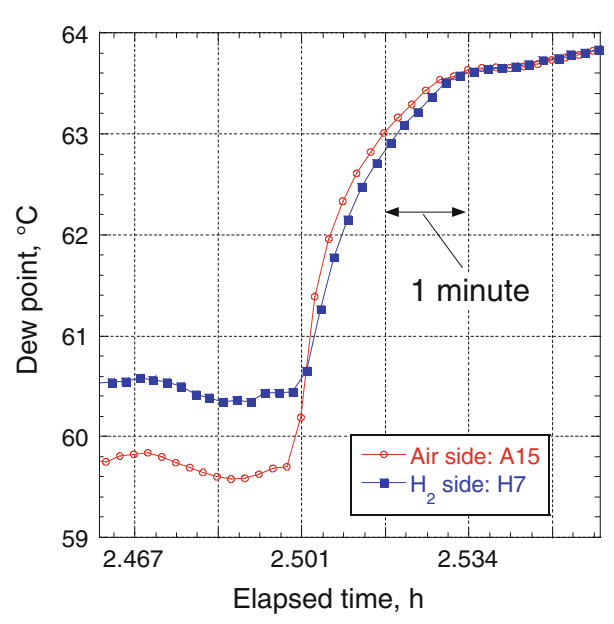

results from $\mathrm{H} 7$ and A15 are shown in Fig. 5). From hour 7 onward, the readings of sensor A4 diverged from the trend of nearby sensors; the cause of this is not known.

There are two striking features of these data that give insight into the operation of this type of fuel cell. The first point concerns the relative values of the dew point for sensors on either side of the MEA, and from sensors distributed around each flow plate. Within the uncertainty of measurement, steady-state data taken from sensors on either side of the MEA are identical. Thus, for example, data from sensors H8 and H9 on the hydrogen (anode) plate correspond closely to data from sensors A1 and A2 from the air (cathode) plate. In contrast, data from sensors along the same channel, on the same side of the MEA, differ significantly. For example, on the hydrogen (anode) plate, data from sensors $\mathrm{H} 8$ and $\mathrm{H} 9$ differ from data from sensors H10 and H12.

The second striking feature of the data concerns the timescales of response. During periods of transition, for example, when the current is first drawn, data from sensors on either side of the MEA show changes in humidity that occur within a few seconds of each other. An example of this is shown for sensors H7 and A15 in Fig. 5.

In addition to humidity transient changes caused by first drawing current, there are other humidity changes observable after several hours of operation, for example, at around $5 \mathrm{~h}$ into the data. These differ in character around the cell active area, showing that changes are localized, and can be spatially resolved using these measurements.

Figure 6 shows a graph of typical pressure sensor data, after calibration correction. The data show significant noise, slow initial stabilization in some cases, and further drift after initial settling. The sensors typically could not be calibrated with the expanded uncertainty better than about $10 \mathrm{kPa}$, and the uncertainty in use is greater than this.

\section{Discussion}

In the fuel cell reaction, water is generated at the air side of the membrane, and this might be expected to predominantly humidify the air, not the hydrogen. However, the 


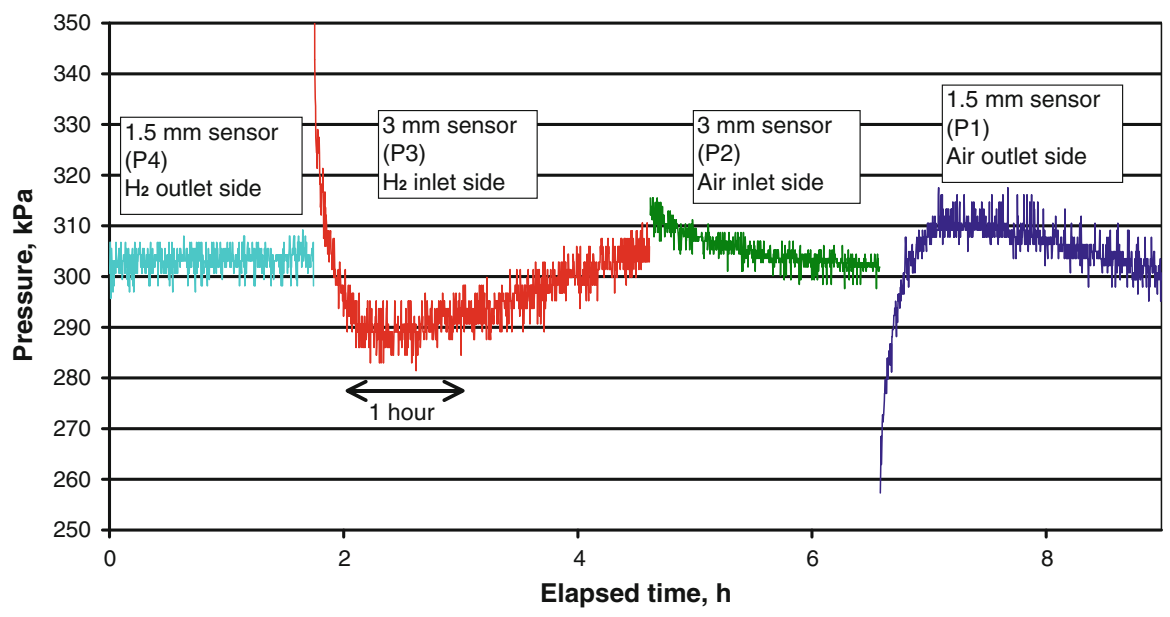

Fig. 6 Example data for miniature pressure sensors embedded in fuel cell plates (calibration correction applied). The applied gas pressure was $0.3 \mathrm{MPa}$, stable to within approximately $0.002 \mathrm{MPa}$. Data are shown for each of the four sensors in turn, including the transient as power is applied to each sensor

results showed the humidity of the hydrogen side to closely match that of the air side, at every location. This suggests that in this experimental configuration moisture freely permeated the MEA.

This observed high level of permeation is in contrast to previous observations for the same MEA. Earlier work on this MEA in a serpentine-flow fuel cell, [6,7] using the same measurement technique, demonstrated clear differences between humidity on the air and hydrogen sides of the MEA. This was explained in terms of two competing mechanisms: electro-osmotic drag drawing water from anode $\left(\mathrm{H}_{2}\right)$ to cathode (air) throughout the current-generating active area of the cell; and back diffusion of water from cathode to anode in regions where the air is more humid than the hydrogen. In the serpentine cell, it was demonstrated that back diffusion dominates electro-osmotic drag [6], leading to increased humidification of the anode gas channel when current is drawn from the cell. In the interdigitated cell, diffusion appeared to be even more dominant, equalizing the humidity across the membrane at all locations on a timescale of seconds or less.

The difference in moisture permeation through the same MEA in different cells is unexpected. Both cells were operated under nominally identical conditions, including the same total flow and transit time for reactant gases. The observed difference must then be attributable to differences in flow pattern. In a serpentine flowfield, gas flows from inlet to outlet uninterrupted, with the GDL being a relatively slow or static region alongside the gas flow. In an interdigitated flowfield, gas flow from inlet channels to outlet channels is forced through the narrow layer of carbon paper GDL. (The term "gas diffusion layer" is a misnomer in this case.) In these flow conditions the GDL is a region of high gas velocity immediately adjoining the membrane. This may contribute to increased mass transfer of water.

This work has focused on developing a measurement capability inside fuel cells. Any conclusions about the fuel cell function are preliminary here, and will be the 
subject of further detailed study. However, some aspects of the results can be immediately interpreted. For example, it can be seen that the humidity near sensors H13 and H14 is relatively low for several hours of operation. This might seem surprising, since this inlet $\mathrm{H}_{2}$ gas arrives via sensors $\mathrm{H} 8$ to $\mathrm{H} 12$ where the humidity is seen to be higher. However, the inlet air across the membrane is relatively dry, and it is concluded that this mainly influences the hydrogen side, via the membrane. A localized lack of water vapor production at this point is taken to signify that little or no current is being generated in this corner of the cell. Towards the end of the $10 \mathrm{~h}$ measurement period, the increased generation of water at this location indicates that this region of the cell eventually begins to actively generate current.

The use in hydrogen of humidity sensors calibrated in air incurs some additional uncertainty due to gas non-ideality (water vapor enhancement factor). In addition, the use in this work of an approximation [6] for conversion of relative humidity to dew point did not fully account for the water vapor enhancement factor. In air at $0.3 \mathrm{MPa}$ at high dew points, the maximum error due to this approximation would be about $1.3 \%$ of the vapor pressure value, corresponding to $0.35^{\circ} \mathrm{C}$ at worst, in these conditions. The water vapor enhancement factor for hydrogen is unknown, but its effect is assumed to be no more than double that for air-corresponding to a dew-point error of $0.7^{\circ} \mathrm{C}$ at worst. For the small between-sensor differences in this work $\left(<10^{\circ} \mathrm{C}\right)$, at near-identical pressures, correlation would be expected to reduce the combined effect to a small fraction of a degree in the dew point. Such an error does not alter the findings of this work, which are not closely dependent on the absolute values but mainly on relative trends between sensors.

The fine-level impact of hydrogen on performance of the humidity sensors is also not known (it does not appear to have a major effect), but similarly if the effect on a given sensor were in the region of $2 \%$ rh or less, the impact on the data differences would again be a fraction of a degree in the dew point, at most.

In principle, in-cell localized pressure measurements are of interest for configurations where the pressure varies progressively through the cell (e.g., serpentine flow), or varies with time. However, the trial of embedded miniature pressure sensors in this work met with only limited success. Most of the sensors used were not capable of calibration or measurement with the required uncertainty, because of sensor drift. The uncertainty due to random noise could be reduced by averaging, and it is possible that a four-wire sensor type would provide a better measurement. Pre-screening would also be advisable to select the best performing sensors.

\section{Conclusion}

In situ temperature, humidity, and pressure measurements within fuel cell channels have been made inside operating hydrogen fuel cells. This work has for the first time extended within-channel measurements to a fuel cell of interdigitated flow channel configuration. In this interdigitated fuel cell, moisture was found to cross the MEA freely, showing it to be functionally transparent to moisture in this setup. This is in contrast to previous observations of the same membrane in a serpentine-channel configuration, where limiting mechanisms maintained a humidity differential across the 
membrane in some parts of the cell. This unexpected difference in the moisture transmission through the MEA appears to be associated with the different gas transport patterns in the two configurations of the gas flowfield.

The humidity and temperature measurements were made successfully in challenging conditions of temperature up to at least $80^{\circ} \mathrm{C}$ and high humidity inside an operating hydrogen PEM fuel cell. The humidity and temperature sensors were sufficiently robust, with adequate spatial and temporal resolution for observing localized changes within different regions of individual channels. The pressure sensors gave only limited useful data, but localized pressure measurements might yield more valuable information, if better sensor performance can be achieved.

Acknowledgments This work was carried out under the Innovation R\&D Programme of the UK Department of Business, Innovation \& Skills. The project was a collaboration with an Industrial Group comprising: Acal Energy, AFC Energy, C Tech Innovation, CMR Fuel Cells, Ceres Power, Imperial College, Intelligent Energy, Johnson Matthey, Rolls Royce Fuel Cell Systems and UCL. The authors are grateful to Sarah Ball and Jonathan Sharman of Johnson Matthey Technology Centre for supply of fuel cell components.

\section{References}

1. S. Tsushima, S. Hirai, Prog. Energy Combust. Sci. 37, 204-220 (2010). doi:10.1016/j.pecs.2010.06. 001

2. M.M. Mench, Q.L. Dong, C.Y. Wang, J. Power Sources 124, 90 (2003)

3. W.P. Partridge, T.J. Toops, J.B. Green, T.R. Armstrong, J. Power Sources 160, 454 (2006)

4. S. Basu, H. Xu, M.W. Renfro, B.M. Cetegen, J. Fuel Cell Sci. Technol. 3, 1 (2006)

5. H. Nishikawa, R. Kurihara, S. Sukemori, T. Sugawara, H. Kobayashi, S. Abe, T. Aoki, Y. Ogami, A. Matsunaga, J. Power Sources 155, 213 (2006)

6. G. Hinds, M. Stevens, J. Wilkinson, M. de Podesta, S. Bell, J. Power Sources 186, 52 (2009)

7. G. Hinds, M. Stevens, J. Wilkinson, M. de Podesta, S. Bell, in Proceedings of Congrés International de Métrologie, Paris, May 2009, CD: ISBN 2-915416-08-7 